\title{
Genetic insights into family group co-occurrence in Cryptocercus punctulatus, a sub-social woodroach from the southern Appalachian Mountains
}

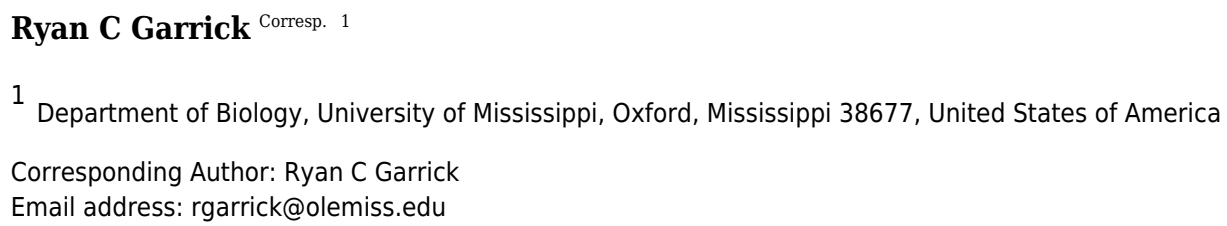

The wood-feeding cockroach Cryptocercus punctulatus Scudder (Blattodea:

Cryptocercidae) is an important member of the dead wood (saproxylic) community in montane forests of the southeastern United States. However, its population biology remains poorly understood. Here, aspects of family group co-occurrence were characterized in order to provide basic information that can be extended by studies into the evolution and maintenance of sub-sociality. Broad sampling across the species' range was coupled with molecular data [mitochondrial DNA (mtDNA) sequences]. The primary questions were: (1) what proportion of rotting logs contain two or more different mtDNA haplotypes and how often can this be attributed to multiple families inhabiting the same log, (2) are multi-family logs spatially clustered, and (3) what levels of genetic differentiation among haplotypes exist within a log, and how genetically similar are matrilines of co-occurring family groups? Multi-family logs were identified on the premise that three different mtDNA haplotypes, or two different haplotypes among adult females, is inconsistent with a single family group founded by one male-female pair. Results showed that of the 88 rotting logs from which multiple adult C. punctulatus were sampled, 41 logs $(47 \%)$ contained two or more mtDNA haplotypes, and at least19 of these logs (22\% overall) were inferred to be inhabited by multiple families. There was no strong evidence for spatial clustering of the latter class of logs. The frequency distribution of nucleotide differences between co-occurring haplotypes was strongly right-skewed, such that most haplotypes were only one or two mutations apart, but more substantial divergences (up to 18 mutations, or $1.6 \%$ uncorrected sequence divergence) do occasionally occur within logs. This work represents the first explicit investigation of family group co-occurrence in C. punctulatus, providing a valuable baseline for follow-up studies. 
1 Genetic insights into family group co-occurrence in Cryptocercus punctulatus,

2 a sub-social woodroach from the southern Appalachian Mountains

3

4 Ryan C. Garrick

5

6 Department of Biology, University of Mississippi, Oxford, MS 38677, USA

7

8 Correspondence: Ryan C. Garrick, Department of Biology, University of Mississippi, Oxford,

9 MS 38677, USA E-mail: rgarrick@olemiss.edu; Tel.: +1-662-915-2341; Fax: +1-662-915-5144

10

11 Running title: Woodroach family group co-occurrence

12

13 Subjects: Entomology; Genetics; Zoology 
14 Abstract. The wood-feeding cockroach Cryptocercus punctulatus Scudder (Blattodea:

15 Cryptocercidae) is an important member of the dead wood (saproxylic) community in montane

16 forests of the southeastern United States. However, its population biology remains poorly

17 understood. Here, aspects of family group co-occurrence were characterized in order to provide

18 basic information that can be extended by studies into the evolution and maintenance of subsociality. Broad sampling across the species' range was coupled with molecular data [mitochondrial DNA (mtDNA) sequences]. The primary questions were: (1) what proportion of rotting logs contain two or more different mtDNA haplotypes and how often can this be attributed to multiple families inhabiting the same log, (2) are multi-family logs spatially clustered, and (3) what levels of genetic differentiation among haplotypes exist within a log, and how genetically similar are matrilines of co-occurring family groups? Multi-family logs were identified on the premise that three different mtDNA haplotypes, or two different haplotypes among adult females, is inconsistent with a single family group founded by one male-female pair. Results showed that of the 88 rotting $\log$ from which multiple adult $C$. punctulatus were sampled, $41 \operatorname{logs}(47 \%)$ contained two or more mtDNA haplotypes, and at least19 of these logs ( $22 \%$ overall) were inferred to be inhabited by multiple families. There was no strong evidence for spatial clustering of the latter class of logs. The frequency distribution of nucleotide differences between co-occurring haplotypes was strongly right-skewed, such that most haplotypes were only one or two mutations apart, but more substantial divergences (up to 18 mutations, or $1.6 \%$ uncorrected sequence divergence) do occasionally occur within logs. This work represents the first explicit investigation of family group co-occurrence in C. punctulatus, providing a valuable baseline for follow-up studies. 


\section{Introduction}

In sub-social invertebrates, offspring stay with parents for extended periods of time, but usually disperse before reproducing themselves (Yip \& Rayor 2014). Numerous studies - particularly those focusing on spiders - have sought to understand how this form of social organization impacts genetic structure within species, and how cooperative group living evolves (e.g., Johannesen et al. 1998; Johannesen \& Lubin 1999; Duncan et al. 2010; Yip et al. 2012). The deepest insights into costs and benefits associated with transitions from sub-sociality to eusociality have been gained by studying closely related lineages that represent different stages along this gradient (Bilde et al. 2005; Helantera et al. 2013). Accordingly, identifying sets of taxa that are suitable for comparative analyses, and characterizing their basic population biology, is of considerable value.

Sub-social Cryptocercus woodroaches are the closest living relatives of extant termites (Lo et al. 2000). As a consequence of the phylogenetic position of Cryptocercus within Blattodea, members of this genus are key evolutionary links for understanding transitions to eusociality (Klass et al. 2008; Nalepa 2015). The best studied members of Cryptocercus are the southern Appalachian Mountain lineages (i.e., the C. punctulatus complex) from the southeastern United

55 States (Bell et al. 2007 and references therein). Yet, owing to their cryptic log-dwelling (saproxylic) nature, little is known about family group formation and co-occurrence in $C$.

57 punctulatus, as direct observation is not possible in most cases. To extend our understanding of the ecological and microevolutionary processes that affect genetic structure and local persistence 59 of sub-social invertebrates, these basic knowledge gaps need to be filled. 
61 Previous work has revealed that C. punctulatus form mate pairs upon reaching maturity, and

62 produce their first — and usually only — clutch of offspring approximately one year later. In

63 addition to excavating galleries and maintaining the nest, parents provide intensive brood care.

64 This includes feeding young instars on hindgut fluids via proctodeal trophallaxis, with care

65 continuing after nymphs become nutritionally independent until the parents die, typically at least

66 three years after the birth of their young (Nalepa 1984, 2015). At this point, offspring are no

67 longer fragile; they are at least half grown and have a robust cuticle (Nalepa \& Grayson 2011).

68 Given that copulation between adults likely takes place within the same gallery of the rotting log

69

in which the pair later raise their family, mate pairs have been considered monogamous since opportunities for extra-pair copulation are few (but see Nalepa \& Grayson 2011). Although the spatial demarcation of family groups is difficult, sampling strategies employed in several molecular studies of C. punctulatus have used a single random sample (e.g., Steinmiller et al. 2001; Aldrich et al. 2004a,b). Depending on the question at hand, this may be adequate.

However, it is worth noting that large logs typically have numerous gallery systems (Nalepa 1984) and so it is possible that multiple genetically divergent family groups do co-occur within a single log. But it is as-yet unknown if this occurs, how often, and from where multiple family groups tend to originate.

\section{Recent work on C. punctulatus at Mountain Lake Biological Station in West Virginia has shed} new light on dispersal and colonization processes, and mate-pair composition. A pitfall trapping study by Nalepa and Grayson (2011) confirmed that large nymphs, sub-adults, and adults do occasionally move between logs. Population genetic studies have repeatedly shown that dispersal 
83 distances of wingless saproxylic invertebrates are often very short (e.g., Sunnucks et al. 2006;

84 Garrick et al. 2007, 2008; Leschen et al. 2008; Marske et al. 2009; Walker et al. 2009; Bull et al.

85 2013), and this is also likely to be true of C. punctulatus (Nalepa et al. 2002). Accordingly,

86 following colonization of an uninhabited log by a male or female woodroach, potential mates

87 probably arrive only from logs within close proximity. This suggests that C. punctulatus mate-

88 pairs may be very close relatives. However, genetic estimates of relatedness between mate-pairs

89 from 36 different $\operatorname{logs}$ at Mountain Lake showed that this is rarely the case (Yaguchi et al. in

90 press). As a group, studies at this particular Biological Station (Nalepa 1984; Nalepa \& Grayson

91 2011; Yaguchi et al. in press) have provided critical baseline data on the population biology $C$.

92 punctulatus, but information about family group co-occurrence, and how this might vary across

93 the species' range, is still lacking.

94

DNA sequence data from maternally-inherited mitochondrial DNA (mtDNA) have enabled inferences about the number and composition of family groups in diverse social animal species (Möller et al. 2001; Faulkes et al. 2003), including insects (Vargo 2003; Holzer et al. 2009). The approach is most powerful when used in conjunction with information on the sex of sampled individuals. In the present study, range-wide geographic sampling of C. punctulatus was coupled with sexing and mtDNA sequencing of multiple adult cockroaches per log to address the following questions: (1) what proportion of rotting logs contain two or more different mtDNA

102 haplotypes and how often can this be attributed to multiple families inhabiting the same $\log ,(2)$ 103 are multi-family logs spatially clustered, and (3) what levels of genetic differentiation among 104 haplotypes exist within a log, and how genetically similar are matrilines of co-occurring family 
105

106

107

108

109

110

111

112

113

114

115

116

117

118

119

120

121

122

123

124

125

126

127

groups? Although these questions focus on basic characteristics of population biology, this work represents the first study to explicitly investigate family group co-occurrence in C. punctulatus.

\section{Taxonomy}

\section{Materials and Methods}

The taxonomic status of southern Appalachian lineages of Cryptocercus is contentious. Briefly, following Kambhampati et al.'s (1996) discovery of four chromosomal races within the group, Burnside et al. (1999) described and named each of them as separate species. However, since no reliable morphological differences were apparent, the only diagnostic characters presented by the authors were mtDNA nucleotides (originally seven species-specific mutations, but subsequently reduced to four by Steinmiller et al. 2001), and the descriptions were based on few reliably classified individuals (i.e., those for which both karyotype and mtDNA sequence were determined). Furthermore, species diagnosis on the basis of mtDNA has been applied inconsistently (e.g., Aldrich et al. 2004a), and the geographic origin of some type material is unclear (Nalepa et al. 2002). Since these issues remain unresolved, the original taxon name, $C$. punctulatus, is used here.

\section{Sampling and rotting log classification}

Adult cockroaches $(n=245)$ were sampled from 88 rotting logs spanning the southern Appalachian Mountains and surrounding areas (Appendix 1), under scientific collecting permits 
128 issued by the Alabama DCNR, Georgia DNR (29-WBH-12-16), USDA Forest Service, and US

129 National Park Service (GRSM-2012-SCI-2242; SHEN-2012-SCI-0015). Sampling involved

130 breaking open the hard outer shell of each log with a hatchet and then carefully dismantling the

131 interior woody material, which usually exhibited advanced-stage brown rot decay, with a small

132 pry bar. Although it was not feasible to follow individual galleries, cockroaches were collected

133 from the same general location within the log, until at least three adults had been caught. In most

134 cases, broods were present with the sampled adults, but were usually not collected. Specimens

135 will be lodged in the University of Mississippi Insect Collection (UMIC) following completion

136 of an on-going project in which they are being used. Two or three adults per $\log (\operatorname{mean}=2.78)$

137 were used for molecular analyses. DNA extraction and polymerase chain reaction amplification,

138 sequencing, alignment and validation of data from mtDNA cytochrome oxidase subunit I (COI)

139 and subunit II (COII) genes followed Garrick (2016), and characteristics of the molecular dataset

140 are summarized in Table 1. For each individual, COI+COII sequences were concatenated (1125-

$141 \mathrm{bp}$ ), and each rotting log was classified as containing cockroaches with the same mtDNA

142 haplotype $v s$. two or more different mtDNA haplotypes (i.e., single-haplotype $v s$. multi-

143 haplotype logs). For the latter group, the sex of each cockroach was determined based on

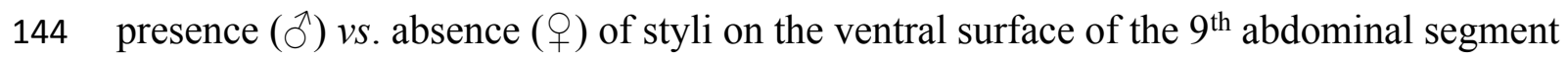

145 (subgenital plate) via examination under 10× magnification. The null hypothesis of a 1:1 sex

146 ratio was then assessed using a $\chi^{2}$ test.

148 The first goal of this study was to determine what proportion of rotting logs contain two or more different mtDNA haplotypes, and how often this can be attributed to multiple families inhabiting

150 the same log. This information would provide new insights into the basic population biology of 
151 C. punctulatus. To achieve this goal, individual-based information on mtDNA haplotype and sex

152 (Appendix 2) was used to distinguish multi-haplotype logs that contained two or more different

153 family groups (i.e., multi-family logs) from those that were consistent with expectations for only

154 a single family (i.e., other multi-haplotype logs). The inference framework was based on the

155 premise that mtDNA is strictly maternally inherited, and non-recombining. Furthermore, it was

156 assumed that de novo mutations are sufficiently rare that their probability of occurrence within

157 the 1125-bp region sequenced in this study was zero. However, it was not assumed that all

158 sampled adults from a given log were from the same age cohort. On this basis, a multi-family log

159 was defined as any log that contained three different haplotypes, or where two females each had

160 a different haplotype (Table 2). All other situations can be attributed to the existence of only a

161 single family derived from a monogamous pair of adults (e.g., via sampling a combination of

162 mother, father, daughters and/or sons), and thus were designated as other multi-haplotype logs.

163 These three log classes (Table 2) were the basis of subsequent analyses.

164

165 Spatial clustering

167 The second goal of this study was to explore whether multi-family logs show a non-random

168 spatial distribution; if so, this might indicate that particular environmental conditions facilitate

169 (or inhibit) family group co-occurrence. For this purpose, Cuzick and Edwards' (1990) test was

170 used to assess the null hypothesis of a random geographic distribution of multi-family logs, as

171 this test can detect global spatial clusters in individual-level data. By treating multi-family logs

172 as cases and assessing clustering relative to controls (i.e., single haplotype logs, other multi-

173 haplotype logs, or both classes combined), this method has the advantage of using only those 
174 geo-spatial coordinates that were actually sampled. The procedure involved calculating the test 175 statistic $T k$ (i.e., the number of cases that neighbor other cases, where $k$ is the number of nearest 176 neighbors to consider) from the empirical data, and then comparing $T k$ to a null distribution

177 generated via Monte Carlo randomizations of case-control labels for each of the spatial locations 178 (9,999 iterations), with significance assessed at the upper tail. Given that the most appropriate 179 neighborhood size is not known a priori, iterations of Cuzick and Edwards' (1990) test was run 180 for values of $k=1$ to 5 , and a Bonferroni correction was used to account for multiple testing. The 181 test was performed for the following data partitions: multi-family logs $(n=19$ cases) $v s$. other 182 multi-haplotype logs $(n=22$ controls $)$, single haplotype logs $(n=47$ controls $)$, or both classes combined ( $n=69$ controls). All spatial clustering analyses were implemented in CLUSTERSEER v2.5.2 (BioMedware, USA). To examine the influence of topography on family group co-occurrence, the null hypothesis of no difference in mean elevation between multifamily logs vs. other multi-haplotype logs, single haplotype logs, or both classes combined, was assessed via two-tailed $t$-tests assuming equal variances (as determined using $F$-tests).

\section{Genetic differentiation among haplotypes}

The third goal of this study was to quantify levels of genetic differentiation among different family group matrilines that co-occur within a $\log$, as this would provide new insights into how sub-socialilty could impact genetic structure within C. punctulatus. By extension, outcomes of this component could point towards potential benefits (or costs) of multi-family living. To quantify levels of genetic divergence among co-occurring haplotypes, MEGA v6.06 (Tamura et al. 2013) was used to calculate the number of nucleotide differences between individuals within 
197 each multi-family and other multi-haplotype log, and these values were then plotted as a

198 frequency distribution. Within logs, redundant haplotypes were omitted, so that only non-zero

199 nucleotide differences were tallied. In the case of multi-family logs, comparisons that could be

200 clearly identified as representing between-family comparisons (e.g., when two co-occurring

201 females each had a different mtDNA haplotype) were partitioned from those that could not (e.g.,

202 when two males and a female each had a different haplotype). To assess whether multi-family vs.

203 other multi-haplotype logs exhibited similar distributions of pairwise nucleotide differences, they

204 were compared via a paired two-sample $t$-test.

205

206

\section{Results}

207

208

\section{Sampling and rotting log classification}

209

210 A total of 155 unique mtDNA haplotypes were identified. Of the 88 rotting logs from which $C$.

211 punctulatus were sampled, 47 (53\%) contained only a single haplotype, whereas $41 \operatorname{logs}$ had two

212 or more different haplotypes. Of the latter group, 19 logs unambiguously contained multiple

213 families (i.e., overall $22 \%$ were multi-family logs), and $22 \operatorname{logs}(25 \%$ overall) were classified as

214 other multi-haplotype logs (Figure 1, Appendix 1). There was a significant female bias in multi-

215 family $\operatorname{logs}\left(71 \%\right.$ females, $n=56$ observations, $\left.\chi^{2}=10.286, d . f .=1, P=0.001\right)$. Conversely,

216 there was no meaningful departure from a 1:1 sex ratio in other multi-haplotype logs (54\%

217 females, $n=116$ observations, $\left.\chi^{2}=0.862, d . f .=1, P=0.353\right)$.

218

219 Spatial clustering 
221 Cuzick and Edwards' (1990) tests showed that there was no clearly detectable departure from the

222 null hypothesis that multi-family logs have a random geographic distribution when the control

223 group was represented by other multi-haplotype logs, or single haplotype logs. Conversely, when

224 single haplotype and other multi-haplotype logs were combined to form the control group, the

225 test statistic was significant after Bonferroni correction $(P=0.045)$. However, only one iteration

226 of the test generated this result (i.e., when a neighborhood size of $k=2$ was assumed). All other

227 neighborhood sizes that were examined (i.e., $k=1,3,4$ and 5) yielded non-significant test

228 statistic values. There was no evidence for elevational partitioning multi-family $v s$. single

229 haplotype logs, other multi-haplotype logs, or both classes combined $(t=0.031$, d.f. $=64, P=$

$2300.975 ; t=-1.042, d . f .=39, P=0.304$; and $t=-0.430, d . f .=86, P=0.668$, respectively).

231

232 Genetic differentiation among haplotypes

233

234 The sequence alignment of 155 unique mtDNA haplotypes contained 308 polymorphic sites,

235 with a maximum 116 nucleotide differences between a pair of haplotypes (i.e., 10.3\%

236 uncorrected sequence divergence). Of the 41 logs with multiple haplotypes, 38 logs contained

237 two, whereas three logs had three different haplotypes. Accordingly, the frequency distribution

238 of nucleotide differences between haplotypes sampled from within the same rotting log was

239 calculated from a total of 47 pairwise comparisons. This frequency distribution showed that

240 while co-occurring haplotypes most often (66\% of the time) differ from one another by only one

241 or two mutations, this is not always the case - modest differences within logs (up to 18

242 mutations, or $1.6 \%$ uncorrected sequence divergence) do also occur (Figure 2). Within multi- 
243 family $\operatorname{logs}$, data points that were not clearly attributable to between-family comparisons were

244 relatively few (20\%; Figure 2). Furthermore, there was no strong discord in the distribution of

245 pairwise nucleotide differences between multi-family $v s$. other multi-haplotype logs $(t=0.410$,

246 d.f. $=17, P=0.343)$.

247

\section{Discussion}

This paper represents the first explicit investigation of family group co-occurrence in $C$.

251

252

253

254

255

256

257

258

259

260

261

262

263

264

265

punctulatus - an evolutionarily important woodroach from a montane forest biodiversity hotspot

(Garrick 2011). Broad geographic sampling was coupled with sequencing of multiple individuals

per rotting in order to address the following questions: (1) what proportion of rotting logs contain two or more different mtDNA haplotypes and how often can this be attributed to multiple families inhabiting the same log, (2) are multi-family logs spatially clustered, and (3) what levels of genetic differentiation among haplotypes exist within a log, and how genetically similar are matrilines of co-occurring family groups? The first question provides insights into an as-yet unknown aspect of the basic population biology of $C$. punctulatus, whereas the second question is exploratory, with the potential to indicate whether environmental factors might influence family group co-occurrence. The third question yields information into how sub-socialilty could impact genetic structure within this species. Together, they provide a framework for subsequent studies. Below, major findings are summarized, and limitations of the present work and recommendations for future studies are also highlighted.

\section{Family group co-occurrence}


267 Approximately half (47\%) of the rotting logs from which C. punctulatus were sampled contained

268 multiple mtDNA haplotypes (Figure 1, Appendix 1). In contrast, Kambhampati et al. (1996)

269 sequenced portions of two mtDNA genes (12S and 16S rRNA; 440-bp; and 406- to 414-bp,

270 respectively) for two C. punctulatus individuals from each of five sites across Virginia, North

271 Carolina, Georgia and Alabama, but found no within-site genetic differences. However, given

272 that the latter study was limited by small sample sizes, it does not provide a strong comparison

273 here. Similarly, whereas the present study found that almost half of the confirmed multi-

274 haplotype logs (22\% overall) clearly contained representatives of two or more family groups

275 (Figure 1, Appendix 1), other molecular studies have routinely pooled C. punctulatus individuals

276 sampled from different logs (e.g., Burnside et al. 1999; Hossain \& Kambhampati 2001) and so

277 they too provide no useable comparative data.

279 One notable exception is a study by Aldrich et al. (2005), who used allozyme markers to screen

280 genetic variation in $\sim 40$ C. punctulatus nymphs from each of 23 sites across the southern

281 Appalachian Mountains. Although samples were often pooled across logs, for 10 sites they were

282

283

284

285

286

287

288 not, and so in these particular cases a single rotting log represented the basic unit of analysis. For this subset of logs, the authors reported that genotype frequencies deviated significantly from Hardy-Weinberg expectations. In nine out of 10 logs the deviation was in the direction of homozygote excess - an outcome that is consistent with Wahlund effect (i.e., genetic substructure, potentially caused by sampling different family groups). However, homozygote excess is also compatible with inbreeding. Notably, recent work by Yaguchi et al. (in press) suggested that mating among close relatives is relatively uncommon in C. punctulatus. Those 
289 authors found that in a sample of 36 mate-pairs genotyped with a set of nuclear microsatellite

290 loci, $72 \%$ were unrelated (the remaining $28 \%$ had parent-offspring, full-sib, or half-sib

291 relationships). Given this, Aldrich et al.'s (2005) data can be explained by frequent sampling of

292 two or more family groups per log, in agreement with conclusions of the present study.

293

294 The finding that multi-family logs are quite common in C. punctulatus is interesting, as there are 295 reasons why single family logs could be advantageous. For example, inhabitants of single family 296 logs would be released from intraspecific competition, including energetically expensive defense 297 of galleries against intruders (Nalepa 2015). Also, high relatedness among all individuals in a 298 single family log could potentially enhance cooperative behaviors (e.g., dislodgment and 299 reduction of dead wood into small particles that can be ingested; Watanabe \& Tokuda 2010), 300 since benefits are received exclusively by kin. However, despite the potential costs of sharing a $301 \log$ with other families, in C. punctulatus this appears to be relatively common.

303 Spatial distribution of multi-family logs

304

Rotting logs that contained representatives of multiple family groups were randomly arrayed 306 across elevational strata, and also across the geographic area that was sampled in this study.

307 Although one of several iterations of Cuzick and Edwards' (1990) test for spatial clustering did 308 indicate that some structure may exist, this outcome was confined to a narrow portion of the 309 parameter space, and so it is generally poorly supported. Overall, the analyses presented here 310 suggest that if there are habitat characteristics that promote (or limit) family group co311 occurrence, they probably operate over finer scales than those examined in this study. 
312 Previously, it has been suggested that large logs may have greater potential to harbor multiple

313 mate-pairs of C. punctulatus than small logs (Yaguchi et al. in press). Log diameter, length, and

314 other microhabitat characteristics that affect saproxylic invertebrates such as decomposition class

315 and moisture content can certainly vary over fine geographic scales in montane areas (e.g.,

316 Barclay et al. 2000; Woodman et al. 2006). Accordingly, an examination of factors that

317 influence family group co-occurrence in C. punctulatus should incorporate local environmental

318 variables. As these data are generally not available at the appropriate resolution from remote

319 sensing and other GIS databases, techniques for measuring very fine-scale ecological data would

320 need to be employed (e.g., Barclay et al. 2000; Grove 2002).

321

322 Genetic differentiation

323

324 The frequency distribution of nucleotide differences between unique haplotypes sampled from

325 the same rotting log was strongly right-skewed, with most co-occurring haplotypes only one or

326 two mutations apart, but with some occurrences of more substantial divergences (up to 18

327 mutations, or 1.6\% uncorrected sequence divergence; Figure 2). Also, there was no strong

328 difference between frequency distributions derived from logs that clearly contained multiple

329 families and those that did not (i.e., multi-family vs. other multi-haplotype logs). This suggests

330 that most co-occurring families share a recent common ancestor. Furthermore, for multi-

331 haplotype logs that potentially contained only a single family (i.e., where a single mate-pair was

332 sampled), the right-skewed frequency distribution indicates that, based on mtDNA data, parental

333 individuals are not all that distantly related. Although Yaguchi et al. (in press) showed that $72 \%$

334 of mate pairs were unrelated (i.e., they shared no more microsatellite alleles per locus than was 
335 expected by chance, given the local frequency of each allele), nuclear genotypic data are most

336 informative over relatively short generation-to-generation timescales. Conversely, DNA

337 sequence data can provide insights over deeper time scales (Sunnucks 2000; Garrick et al. 2006,

338 2010, 2015). For instance, mate-pairs that are second cousins would probably not register as

339 being related on the basis of a small set of microsatellite loci due to several generations of

340 gametic recombination, but they could nonetheless be identified as close relatives on the basis of

341 their more slowly evolving mtDNA haplotype. Thus, the notion that co-occurring families share

342 a recent common ancestor is not incompatible with Yaguchi et al.'s (in press) findings.

344 Generally speaking, high genetic diversity among individuals that co-occur at a site promotes

345 long-term persistence of the local population (Frankham 2005, and references therein). However,

346 in circumstances where antagonistic and competitive intraspecific interactions mostly occur

347 among non-siblings, high within-site relatedness may be advantageous (Caesar et al. 2010).

348 Baseline data from the present study indicate that compared to genetically polymorphic single

349 family logs, there is no meaningful increase in within-site mtDNA-based diversity among $C$.

350 punctulatus that share their rotting log with other families. Although mtDNA sequence

351 divergence may be only loosely correlated with genome-wide nuclear genetic diversity (Zhang \&

352 Hewitt 2003), the empirical data nonetheless suggest that if there are benefits to living in a multi-

353 family log, increased local genetic diversity is unlikely to contribute to this.

355 In addition to characterizing aspects of family group co-occurrence for the purpose of

356 understanding sub-sociality, the present study also provides an opportunity to examine the

357 adequacy of randomly sampling a single C. punctulatus individual per rotting log (e.g., 
358 Steinmiller et al. 2001; Aldrich et al. 2004a,b). Based on levels of genetic differentiation among

359 co-occurring haplotypes seen here, the one-sample-per-log strategy would fail to fully capture

360 within-log diversity about half of the time. Even though the majority of co-occurring haplotypes

361 have few mutational differences, some divergences are more substantial (Figure 2). Indeed, $C$.

362 punctulatus from highly divergent genetic lineages - including those that likely differ in

363 chromosome number - may occasionally co-inhabit the same log (Garrick 2016). Accordingly,

364 depending on the goals of the study, it may be prudent to sample multiple individuals from a log.

\section{Limitations and future directions}

Several limitations of this study warrant consideration. First, while the overall sample size and geographic coverage was large, sample sizes per log were quite small. Accordingly, it was not possible to have a confirmed absence of multiple families (or haplotypes) within a given log. test assumes that controls were correctly classified. This also means that the reported frequencies

373 of co-occurrence of multiple haplotypes ( $47 \%$ of logs) and family groups (22\% of logs) represent

374 lower bounds, not point estimates. This consideration may partly reconcile the much higher

375 frequency of multi-family logs (90\% of relevant logs) suggested by Aldrich et al.'s (2005)

376 allozyme data. However, testing of Hardy-Weinberg equilibrium is not an ideal framework for

377 making inferences about family group co-occurrence as there are several possible causes for 378 departures from expected genotype frequencies, and so Aldrich et al.'s (2005) data may provide 379 an overestimate. A second limitation of the present study is that the mtDNA-based inference 380 framework has a reduced ability to detect family group co-occurrence in logs from which few 
381 females are sampled (e.g., a significantly female-biased sex ratio was detected within multi-

382 family logs, but not within other multi-haplotype logs; also see Table 1). This means that

383 resolution of the approach used here may not be equal across all logs. Third, the simplifying

384 assumption of no de novo mutations may not hold true. However, the mtDNA mutation rate

385 would need to be exceptionally high to overturn inferences of multiple families within a log, and

386 so this limitation is a source of noise (cf. positively misleading).

387

388 For understanding family group co-occurrence in C. punctulatus, neither a mtDNA-only

389 approach, nor a nuclear marker-only approach, is flawless. The clearest insights would be gained

390 by coupling genotypic data from multiple bi-parentally inherited nuclear markers with haplotypic

391 data from maternally inherited mtDNA, together with information on the sex (and also age

392 cohort) of each individual. Dense sampling — without pooling across logs — is also important, and

393 it would be beneficial to quantify characteristics of the log itself so that potential influences of

394 microhabitat on family group co-occurrence could be investigated. Thus, the present study

395 represents a baseline against which follow-up studies can be compared, and the current findings

396 should be considered a working hypothesis, to be refined with additional data.

397

398

399

400

401

402

\section{Acknowledgments}

Sequencing was performed by staff at Yale University’s DNA Analysis Facility on Science Hill.

R.J. Worthington assisted with cockroach sex identifications, R.J. Dyer, C.Q. Hyseni and R.E.

Symula assisted with some fieldwork, and R.N. Yi contributed to some laboratory work. This 
403 paper also benefited from constructive comments from D.P.W. Huber and two anonymous

404 reviewers.

405

406

\section{References}

407

408

Aldrich BT, Krafsur E, Kambhampati S (2004a) Species-specific allozyme markers for

409

Appalachian wood-feeding cockroaches (Dictyoptera: Cryptocercidae). Biochemical Genetics,

410

42, 149-164.

411

412

Aldrich BT, Zolnerowich G, Kambhampati S (2004b) Interspecific morphological variation in 413 the wood-feeding cockroach, Cryptocercus (Dictyoptera: Cryptocercidae). Arthropod Structure

414 and Development, 33, 443-451.

415

416

Aldrich BT, Kambhampati S, Krafsur ES (2005) Population genetics of wood-feeding cockroaches in the genus Cryptocercus. Journal of Heredity, 96, 572-575.

Barclay S, Ash JE, Rowell DM (2000) Environmental factors influencing the presence and abundance of a log-dwelling invertebrate, Euperipatoides rowelli (Onychophora:

Peripatopsidae). Journal of the Zoological Society of London, 250, 425-436.

Bell WJ, Roth LM, Nalepa CA (2007) Cockroaches: Ecology, Behavior, and Natural History. 
426 Bilde T, Lubin Y, Smith D, Schneider JM, Maklakov AA (2005) The transition to social inbred

427 mating systems in spiders: Role of inbreeding tolerance in a subsocial predecessor. Evolution, $42859,160-174$.

429

430

Bull, JK, Sands CJ, Garrick RC, Gardner MG, Tait NN, Briscoe DA, Rowell DM, Sunnucks P

431

(2013) Environmental complexity and biodiversity: The multi-layered evolutionary history of a

432

log-dwelling velvet worm in montane temperate Australia. PLoS ONE, 8, e84559.

433

434

Burnside CA, Smith PT, Kambhampati S (1999) Three new species of the wood roach,

Cryptocercus (Blattodea: Cryptocercidae), from the Eastern United States. Journal of the Kansas

Entomological Society, 72, 361-378.

437

Caesar S, Karlsson M, Forsman A (2010) Diversity and relatedness enhance survival in colour polymorphic grasshoppers. PLOS ONE, 5, e10880.

Cuzick J, Edwards R (1990) Spatial clustering for inhomogeneous populations. Journal of the Royal Statistical Society Series B, 52, 73-104.

443

444

Duncan SI, Riechert SE, Fitzpatrick BM, Fordyce JA (2010) Relatedness and genetic structure in 445 a socially polymorphic population of the spider Anelosimus studiosus. Molecular Ecology, 19, $446 \quad 810-818$. 
448 Faulkes CG, Arruda MF, Monteiro Da Cruz MAO (2003) Matrilineal genetic structure within 449 and among populations of the cooperatively breeding common marmoset, Callithrix jacchus. 450 Molecular Ecology, 12, 1101-1108.

451

452 Frankham R (2005) Genetics and extinction. Biological Conservation, 126, 131-140.

453

454 Garrick RC (2011) Montane refuges and topographic complexity generate and maintain 455 invertebrate biodiversity: Recurring themes across space and time. Journal of Insect 456 Conservation, 15, 469-478.

457

458 Garrick RC (2016) True syntopy between chromosomal races of the Cryptocercus punctulatus 459 Scudder (Blattodea: Cryptocercidae) wood-roach species complex. Insectes Sociaux, 3, 353-355. 460

461 Garrick RC, Caccone A, Sunnucks P (2010) Inference of population history by coupling 462 exploratory and model-driven phylogeographic analyses. International Journal of Molecular 463 Sciences, 11, 1190-1227.

464

465 Garrick RC, Kajdacsi B, Russello MA, Benavides E, Hyseni C, Gibbs JP, Tapia W, Caccone A 466 (2015) Naturally rare versus newly rare: Demographic inferences on two timescales inform 467 conservation of Galápagos giant tortoises. Ecology and Evolution, 5, 676-694. 
469 Garrick RC, Rowell DM, Simmons CS, Hillis DM, Sunnucks P (2008) Fine-scale

470 phylogeographic congruence despite demographic incongruence in two low-mobility saproxylic

471 springtails. Evolution, 62, 1103-1118.

472

473 Grove SJ (2002) Tree basal area and dead wood as surrogate indicators of saproxylic insect

474 faunal integrity: A case study from the Australian lowland tropics. Ecological Indicators, 1, 171475188.

476

477 Helantera H, Aehle O, Roux M, Heinze J, D'Ettorre P (2013) Family-based guilds in the ant

478 Pachycondyla inversa. Biology Letters, 9, 20130125.

479

480

Holzer B, Keller L, Chapuisat M (2009) Genetic clusters and sex-biased gene flow in a

481 unicolonial Formica ant. BMC Evolutionary Biology, 9, 69.

482

483

Hossain S, Kambhampati S (2001) Phylogeny of Cryptocercus species (Blattodea:

484 Cryptocercidae) inferred from nuclear ribosomal DNA. Molecular Phylogenetics and Evolution, $48521,162-165$.

486

487 Johannesen J, Baumann T, Seitz A, Veith M (1998) The significance of relatedness and gene

488 flow on population genetic structure in the subsocial spider Eresus cinnaberinus (Araneae:

489

Eresidae). Biological Journal of the Linnean Society, 63, 81-98.

490 
491 Johannesen J, Lubin Y (1999) Group founding and breeding structure in the subsocial spider

492 Stegodyphus lineatus (Eresidae). Heredity, 82, 677-686.

493

494 Kambhampati S, Luykx P, Nalepa CA (1996) Evidence for sibling species in Cryptocercus

495 punctulatus, the wood roach, from variation in mitochondrial DNA and karyotype. Heredity, 76, $496 \quad 485-496$.

497

498 Klass K-D, Nalepa C, Lo N (2008) Wood-feeding cockroaches as models for termite evolution

499 (Insecta: Dictyoptera): Cryptocercus vs. Parasphaeria boleiriana. Molecular Phylogenetics and 500 Evolution, 46, 809-817.

501

502

Leschen RAB, Buckley TR, Harman HM, Shulmeister J (2008) Determining the origin and age 503 of the Westland beech (Nothofagus) gap, New Zealand, using fungus beetle genetics. Molecular 504 Ecology, 17, 1256-1276.

505

506 Marske KA, Leschen RAB, Barker GM, Buckley TR (2009) Phylogeography and ecological 507 niche modelling implicate coastal refugia and trans-alpine dispersal of a New Zealand fungus 508 beetle. Molecular Ecology, 18, 5126-5142.

509

510 Möller LM, Beheregaray LB, Harcourt RG, Krützen M (2001) Alliance membership and kinship

511 in wild male bottlenose dolphins (Tursiops aduncus) of southeastern Australia. Proceedings of

512 the Royal Society B: Biological Sciences, 268, 1941-1947. 
514 Nalepa CA (1984) Colony composition, protozoan transfer and some life history characteristics

515 of the woodroach Cryptocercus punctulatus Scudder (Dictyoptera: Cryptocercidae). Behavioral

516 Ecology and Sociobiology, 14, 273-279.

517

518 Nalepa CA (2015) Origin of termite eusociality: Trophallaxis integrates the social, nutritional, 519 and microbial environments. Ecological Entomology, 40, 323-335.

520

521 Nalepa CA, Grayson KL (2011) Surface activity of the xylophagous cockroach Cryptocercus

522 punctulatus (Dictyoptera: Cryptocercidae) based on collections from pitfall traps. Annals of the

523 Entomological Society of America, 104, 364-368.

524

525 Nalepa CA, Luykx P, Klass K-D, Deitz LL (2002) Distribution of karyotypes of the

526 Cryptocercus punctulatus species complex (Dictyoptera: Cryptocercidae) in the Southern

527 Appalachians: Relation to habitat and history. Annals of the Entomological Society of America, $52895,276-287$.

529

530 Steinmiller B, Kambhampati S, Brock BL (2001) Geographic distribution of, and genetic

531 variation in, the wood roach Cryptocercus (Dictyoptera: Cryptocercidae) in the United States.

532 Annals of the Entomological Society of America, 94, 732-742.

533

534 Sunnucks P (2000) Efficient genetic markers for population biology. Trends in Ecology and 535 Evolution, 15, 199-203.

536 
537 Sunnucks P, Blacket MJ, Taylor JM, Sands CJ, Ciavaglia SA, Garrick RC, Tait NN, Rowell DM,

538 Pavlova A (2006) A tale of two flatties: Different responses of two terrestrial flatworms to past

539 environmental climatic fluctuations at Tallaganda in montane southeastern Australia. Molecular

540 Ecology, 15, 4513-4531.

541

542 Tamura K, Stecher G, Peterson D, Filipski A, Kumar S (2013) MEGA6: Molecular Evolutionary

543 Genetics Analysis version 6.0. Molecular Biology and Evolution, 30, 2725-2729.

544

545 Vargo EL (2003) Hierarchical analysis of colony and population genetic structure of the eastern

546 subterranean termite, Reticulitermes flavipes, using two classes of molecular markers. Evolution,

$547 \quad 57,2805-2818$.

548

549 Walker MJ, Stockman AK, Marek PE, Bond JE (2009) Pleistocene glacial refugia across the 550 Appalachian Mountains and coastal plain in the millipede genus Narceus: Evidence from

551 population genetic, phylogeographic, and paleoclimatic data. BMC Evolutionary Biology, 9, 25.

552

553 Watanabe H, Tokuda G (2010) Cellulolytic systems in insects. Annual Review of Entomology, $55455,609-632$.

555

556 Woodman JD, Ash JE, Rowell DM (2006) Population structure in a saproxylic funnelweb spider 557 (Hexathelidae: Hadronyche) along a forested rainfall gradient. Journal of Zoology, 268, 325558333.

559 
560 Yaguchi H, Hayashi Y, Tohoku T, Nalepa C, Maekawa K (in press) Genetic data indicate that

561 most field-collected woodroach pairs are unrelated. Insect Science.

562

563 Yip EC, Rayor LS (2014) Maternal care and subsocial behaviour in spiders. Biological Reviews, $56489,427-449$.

565

566 Yip EC, Rowell DM, Rayor LS (2012) Behavioural and molecular evidence for selective

567 immigration and group regulation in the social huntsman spider, Delena cancerides. Biological

568 Journal of the Linnean Society, 106, 749-762.

569

570 Zhang D-X, Hewitt GM (2003) Nuclear DNA analyses in genetic studies of populations:

571 Practice, problems and prospects. Molecular Ecology, 12, 563-584. 
Figure 1

Map showing spatial distributions of classified rotting logs

Figure 1. Map of the southeastern USA showing spatial distributions of rotting logs classified as single haplotype (green circle), multi-family (red star), or other multi-haplotype (blue diamond) logs, based on mtDNA sequence data coupled with information on the sex of $C$. punctulatus individuals. State abbreviations are: Alabama, AL; Georgia, GA, Kentucky, KY; North Carolina, NC; South Carolina, SC: Tennessee, TN; Virginia, VA; and West Virginia, WV.

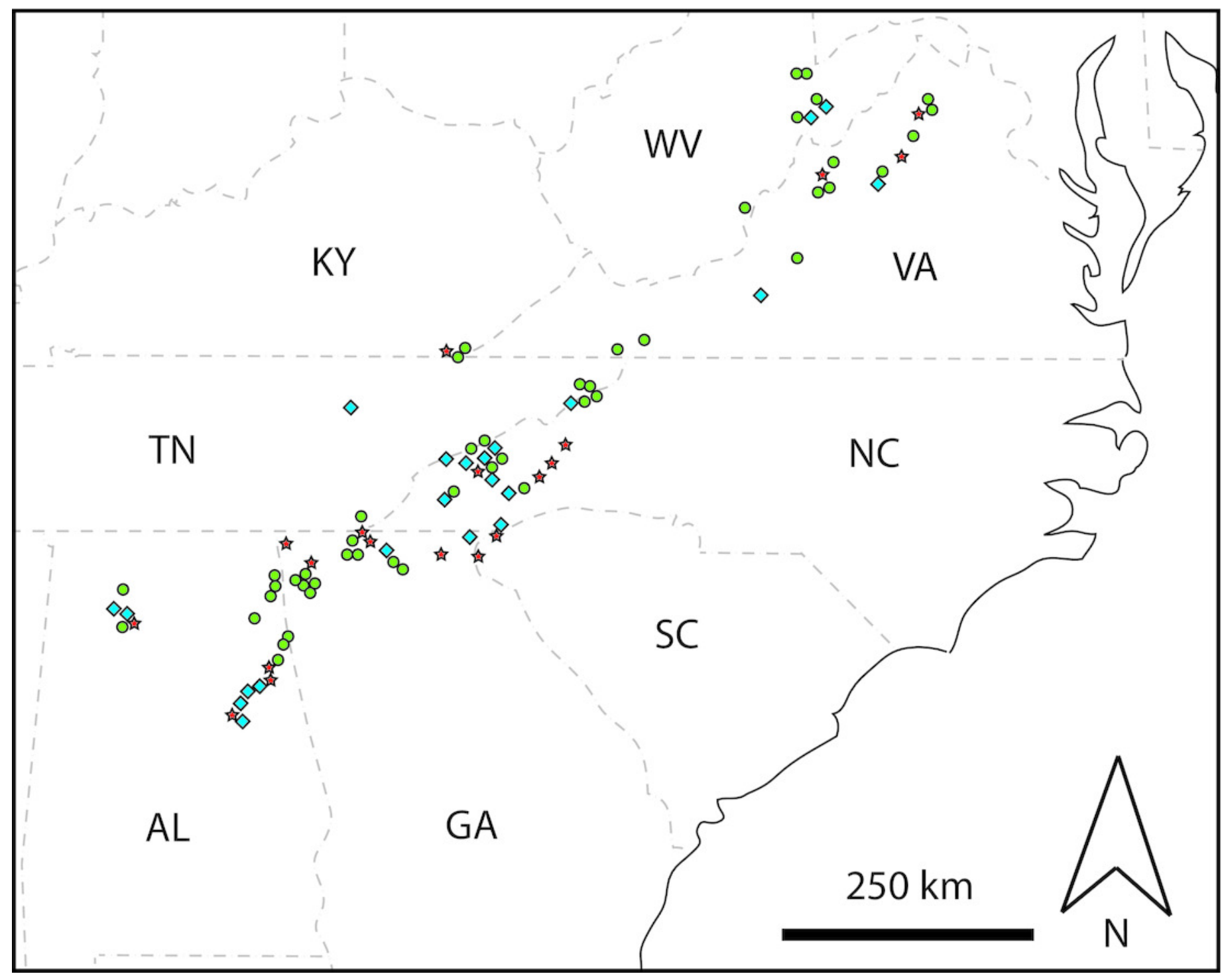




\section{Figure 2}

Frequency distribution of the number of nucleotide differences between pairs of nonredundant mtDNA haplotypes from $C$. punctulatus individuals sampled from the same rotting log.

Figure 2. Frequency distribution of the number of nucleotide differences between pairs of non-redundant mtDNA haplotypes from $C$. punctulatus individuals sampled from the same rotting log. Shading of bars represents the proportional contribution of multi-family (black plus grey) vs. other multi-haplotype (white) logs to the overall tally. The bi-partitioning of multi-family logs delineates comparisons that could (black squares) vs. could not (pale grey) be clearly identified as representing between-family comparisons.

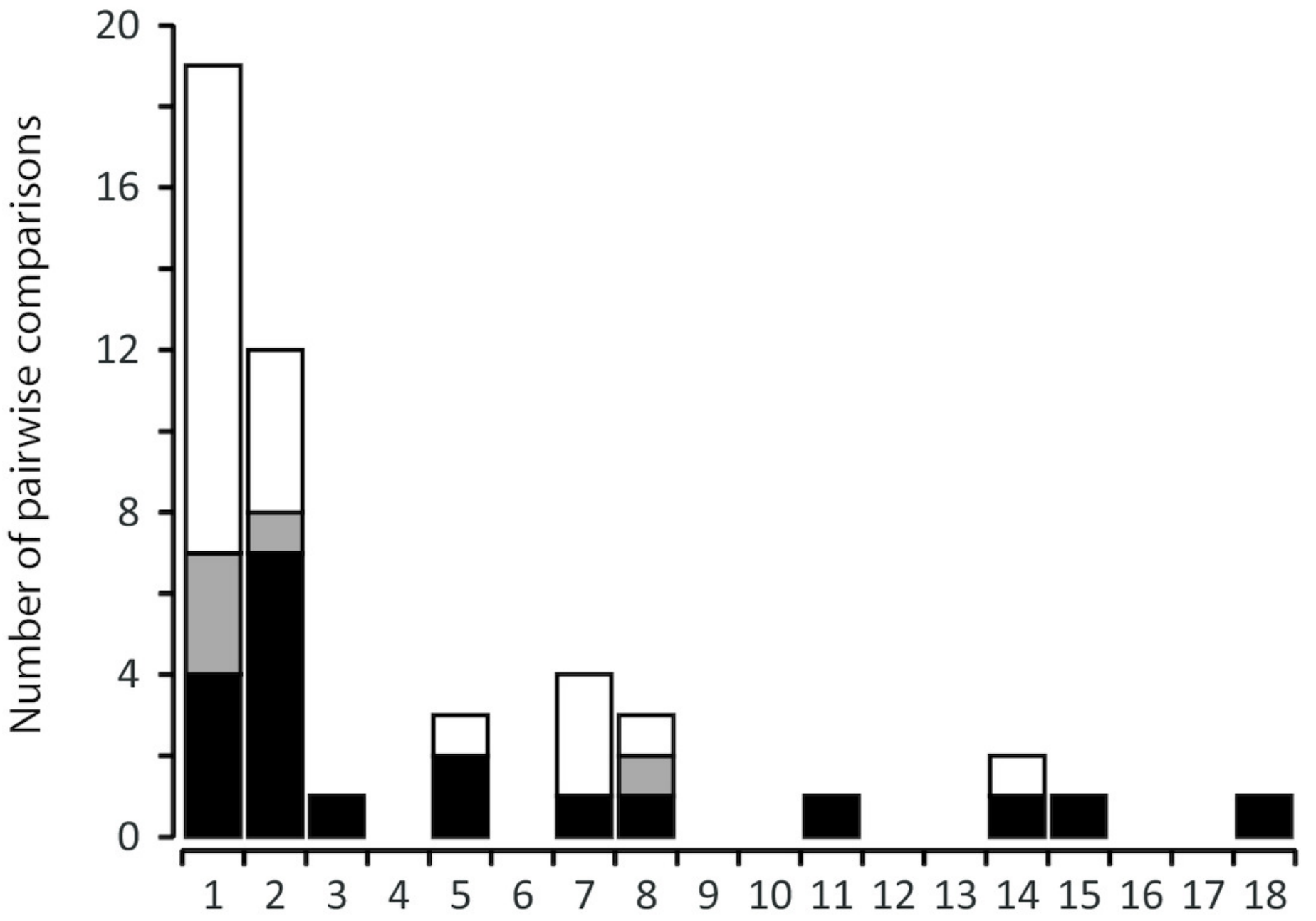




\section{Table $\mathbf{1}$ (on next page)}

Characteristics of mtDNA sequence data

Table 1. Characteristics of mtDNA sequence data generated from 245 C. punctulatus individuals sampled in the present study. Summary statistics are presented for each gene separately, and also for the concatenated dataset. Abbreviations are as follows: number of base pairs, bp; proportion of guanine plus cytosine nucleotides, $\mathrm{G}+\mathrm{C} \%$; nucleotide composition maximum-likelihood estimate ( $G T R+I+G$ model) of transition / transversion ratio, ts/tv; number of segregating sites, $S$; and number of unique haplotypes, $N_{\text {hap }}$. 
1

\begin{tabular}{|c|c|c|c|c|c|c|}
\hline $\begin{array}{l}\text { mtDNA gene } \\
\text { region }\end{array}$ & $\begin{array}{l}\text { Alignment } \\
\text { length (bp) }\end{array}$ & $\mathrm{G}+\mathrm{C} \%$ & ts/tv & $S$ & $N_{\text {hap }}$ & $\begin{array}{c}\text { GenBank } \\
\text { accessions }\end{array}$ \\
\hline $\mathrm{COI}$ & 762 & 34.4 & 4.2 & 199 & 142 & $\begin{array}{l}\text { KX944872 - } \\
\text { KX945114; and } \\
\text { KU609620 - } \\
\text { KU609621 }\end{array}$ \\
\hline COII & 363 & 25.7 & 6.7 & 109 & 64 & $\begin{array}{l}\text { KX945115 - } \\
\text { KX945357; and } \\
\text { KU609623 - } \\
\text { KU609624 }\end{array}$ \\
\hline Concatenated & 1125 & 33.8 & 4.9 & 308 & 155 & \\
\hline
\end{tabular}

2 


\section{Table 2 (on next page)}

Hypothetical combinations of mtDNA haplotype $x$ individual sex, for a rotting log from which three adults were sampled

Table 2. All possible hypothetical combinations of mtDNA haplotype $\times$ individual sex, for a rotting log from which three adults were sampled. For each row, different typeface (italics, underlined, or normal) represents different mtDNA haplotypes among individuals within a log, $M$ indicates a male (i.e., father or son), and $F$ indicates a female (i.e., mother or daughter). Multi-haplotype logs that unambiguously contain multiple families are labeled MF, whereas those which are consistent with the existence of only a single family are labeled $\mathrm{OMH}$. Logs that contain a single mtDNA haplotype are designated $\mathrm{SH}$. 


\begin{tabular}{|c|c|c|c|c|}
\hline $\begin{array}{c}\text { No. of different mtDNA } \\
\text { haplotypes }\end{array}$ & Individual 1 & Individual 2 & Individual 3 & $\begin{array}{l}\text { Rotting log } \\
\text { classification }\end{array}$ \\
\hline 1 & $\underline{\mathrm{M}}$ & $\underline{\mathrm{M}}$ & $\underline{\mathrm{M}}$ & $\mathrm{SH}$ \\
\hline 2 & $\underline{\mathrm{M}}$ & $\mathrm{M}$ & $\mathrm{M}$ & $\mathrm{OMH}$ \\
\hline 3 & $\underline{\mathrm{M}}$ & $\mathrm{M}$ & $M$ & $\mathrm{MF}$ \\
\hline 1 & $\underline{F}$ & $\underline{F}$ & $\underline{F}$ & $\mathrm{SH}$ \\
\hline 2 & $\underline{F}$ & $\mathrm{~F}$ & $\mathrm{~F}$ & $\mathrm{MF}$ \\
\hline 3 & $\underline{F}$ & $\mathrm{~F}$ & $F$ & $\mathrm{MF}$ \\
\hline 2 & $\underline{\mathrm{M}}$ & $\mathrm{F}$ & $\mathrm{F}$ & $\mathrm{OMH}$ \\
\hline 2 & $\underline{\mathrm{M}}$ & $\underline{F}$ & $\mathrm{~F}$ & $\mathrm{MF}$ \\
\hline 1 & $\underline{\mathrm{M}}$ & $\underline{F}$ & $\underline{F}$ & $\mathrm{SH}$ \\
\hline 3 & $\underline{\mathrm{M}}$ & $F$ & $\mathrm{~F}$ & $\mathrm{MF}$ \\
\hline 2 & $\underline{\mathrm{M}}$ & $\mathrm{M}$ & $\mathrm{F}$ & $\mathrm{OMH}$ \\
\hline 2 & $\underline{\mathrm{M}}$ & $\underline{\mathrm{M}}$ & $\mathrm{F}$ & $\mathrm{MF}$ \\
\hline 1 & $\overline{\mathrm{M}}$ & $\underline{\mathrm{M}}$ & $\underline{F}$ & $\mathrm{SH}$ \\
\hline 3 & $\underline{M}$ & $M$ & $\mathrm{~F}$ & $\mathrm{MF}$ \\
\hline
\end{tabular}

1 